\title{
Genetic Covariance Structure of Incisor Crown Size in Twins
}

\author{
P.J. Dempsey ${ }^{1}$, G.C. Townsend ${ }^{1}$, N.G. Martin ${ }^{2}$, and M.C. Neale ${ }^{3}$ \\ 'Department of Dentistry, University of Adelaide, South Australia, Australia 5005; 2Queensland Institute of Medical Research, $300 \mathrm{Herston}$ \\ Rd., Herston, Queensland, Australia 4029; '3 Department of Psychiatry, Medical College of Virginia, Box 710, Richmond, Virginia, USA 23298- \\ 0710; "corresponding author
}

\begin{abstract}
Previous studies of tooth size in twins and their families have suggested a high degree of genetic control, although there have been difficulties separating the various genetic and environmental effects. A genetic analysis of variation in crown size of the permanent incisors of South Australian twins was carried out, with structural equation modeling used to determine the relative contributions of genetic and environmental factors. Maximum mesiodistal crown dimensions of maxillary and mandibular permanent incisors were recorded from dental models of 298 pairs of twins, including 149 monozygous (MZ) and 149 dizygous (DZ) pairs. The analysis revealed that: (i) an adequate fit required additive genetic and unique environmental components; (ii) augmenting the model with non-additive genetic variation did not lead to a significant improvement in fit; (iii) there was evidence of shared environmental influences in the upper central incisors of males; (iv) the additive genetic component constituted a general factor loading on all eight teeth, with group factors loading on antimeric pairs of teeth; ( $v$ ) unique environmental effects were mostly variable-specific; (vi) most factor loadings on antimeric tooth pairs could be constrained to be equal, indicating a symmetry of genetic and environmental influences between left and right sides; and (vii) estimated heritability of the incisor mesiodistal dimensions varied from 0.81 to 0.91 .
\end{abstract}

Key words: tooth size, twins, genetics, environment.

\section{Introduction}

Tooth size in human populations has been the subject of numerous investigations to determine, among other aspects, the patterns of variability of different teeth, associations within and between the dental arches, and the relative degrees of influence of genetic and environmental factors. The findings have been reviewed recently by Kieser (1990) and Lauweryns et al. (1993). An examination of within-pair differences in mesiodistal and buccolingual crown dimensions in 75 pairs of twins provided evidence for (i) strong genetic control of individual crown dimensions, (ii) the existence of independent genes or groups of genes contributing to variability in mesiodistal and buccolingual dimensions, and (iii) independent genetic determination of maxillary and mandibular teeth (Potter et al., 1976). In fact, most evidence points to mesiodistal and buccolingual crown dimensions being to the largest extent genetically determined (e.g., Alvesalo and Tigerstedt, 1974; Garn, 1977). Although morphogenetic field theory (Butler, 1939; Dahlberg, 1945) implies that there should be distinct patterns of heritability within each tooth class (incisor, premolar, and molar), results of previous investigations in twins and siblings have been inconsistent (e.g., Lundström, 1948; Alvesalo and Tigerstedt, 1974; Mizoguchi, 1977).

Unfortunately, most previous studies of the dentition do not provide estimates of the role of common or family environment, maternal effects, interaction between genes (epistasis), or genotype-environment interaction, and so the estimates of heritability obtained probably represent the upper limit to the true values. Modern approaches to estimating heritability involve structural equation modeling, which allows hypotheses regarding the relative contributions of genetic and environmental influences to the variation within, and covariation between, variables to be tested (Jöreskog, 1973; Martin and Eaves, 1977; Heath et al., 1989; Neale and Cardon, 1992). Briefly, linear structural equation models are fitted to raw data or summary covariance or correlation matrices by maximum likelihood 


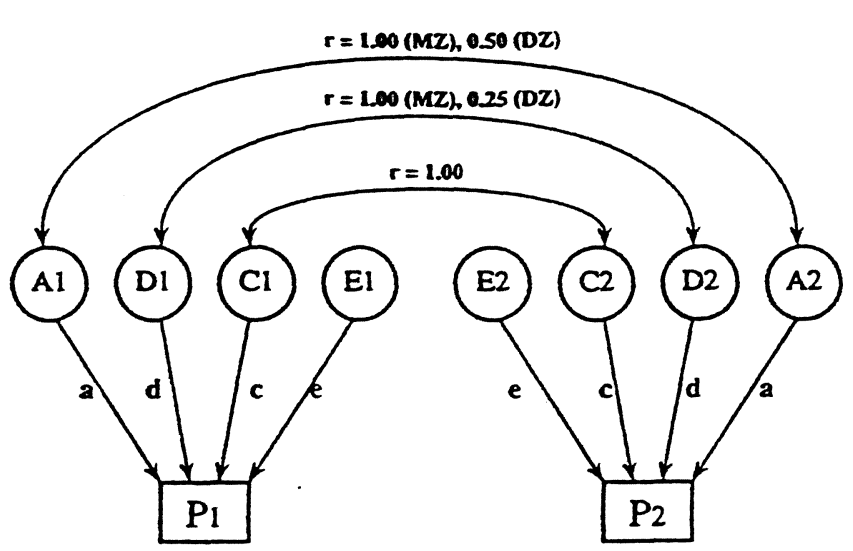

Figure 1. Univariate path diagram showing four potential influences affecting the phenotypes of $M Z$ and $D Z$ twin pairs. $\mathrm{Pl}$ and $P 2$ represent the phenotypes of the first and second twin pair members, respectively. The latent factors $A, D, C$, and $E$ denote the additive genetic variation, non-additive genetic variation, common environmental variation, and unique environmental variation, respectively, for each twin. The double-headed arrows indicate the correlations $(r)$ between latent factors in co-twins. The path coefficients, a, d, c, and $e$, indicate the relative importance of each of the contributing influences, A, D, C, and E (from Neale and Cardon, 1992).

or other methods. Models may incorporate additive (A) and non-additive (D) genetic variation, and shared (C) and random (E) environmental effects, or a subset of these parameters. Along with efficient parameter estimates, the method provides a test of goodness-of-fit to the data. In one such investigation of Pima Indian families, it was estimated that $35 \%$ of the variance in the lateral incisor mesiodistal dimension was due to genetic and environmental transmissible (shared) factors, considerably less than that proposed in earlier studies (Potter et al., 1983).

The aim of this investigation was to analyze the covariance structure of the mesiodistal dimensions of permanent incisor crowns in 298 pairs of South Australian twins, to quantify the relative contributions of genetic and environmental factors to each incisor, and to test whether the size of each tooth was determined independently. Heritabilities were also calculated and examined to see if they supported the predictions of Butler's field theory with respect to the central and lateral incisors.

\section{Materials and methods}

\section{Study population and measurement methods}

Alginate dental impressions were obtained from 82 female $M Z$ and 67 male $M Z$ twin pairs, 48 female $D Z$ and 44 male $D Z$ pairs, and 57 opposite-sexed $D Z$ pairs. The twins ranged in age from 6 to 62 years, although $90 \%$ were between 10 and 25 years old, and the mean age was 16.5 years. Corrections for age were not considered necessary, since the final sizes of dental crowns are determined before emergence of the teeth into the oral cavity, and since measurements were precluded where there was any evidence of attrition affecting the dimension. Zygosities were confirmed by examination of the blood antigens ABO, Rh, MNS,
$\mathrm{Jk}$, and $\mathrm{Fy}$, as well as serum enzyme polymorphisms $\mathrm{ACP}, \mathrm{AK} 1$, ESD, GLO, GPT, PGD, PGM1, and PGP, and protein polymorphisms GC, HP, $\mathrm{Pi}$, and $\mathrm{C} 3$. The probability of dizygosity, given concordance for all systems, was less than $1 \%$. Facial photographs and fingerprints provided confirmatory evidence of zygosity status. Data collection methods were approved by the Committee on the Ethics of Human Experimentation, University of Adelaide (Approval No. $\mathrm{H} / 07 / 84$ ), and all participants were informed volunteers.

Using stone models prepared from the impressions, we measured the maximum mesiodistal and buccolingual crown diameters (following Moorrees et al., 1957) from all emerged and sufficiently intact permanent teeth, except the third molars. The measuring equipment was comprised of sharpened Mitutoyo digital vernier calipers, connected through a multiplexer unit to an Apple IIC microcomputer. To estimate the reliability of the measurement procedure, two investigators measured 50 models independently. Although 56 dimensions were measured, the results of genetic analyses for only the mesiodistal dimensions of the eight permanent incisors are reported here.

\section{Statistical methods}

Mean values and standard deviations were calculated for male and female twins for each variable. Student's $t$ tests were performed for comparison of mean values between sexes, zygosity groups, and first- and second-born twins. Male and female variances were compared by the variance ratio $(F)$ test. Pearson correlation coefficients were calculated between all pairs of teeth, and between twins for each tooth.

Before proceeding with modeling of covariance structure, we explored the data to test for any genotype-by-environment (GXE) interaction, and to determine the likelihood of detecting any non-additive genetic variation that may have existed. The presence of GXE interactions may be indicated by significant regression of $M Z$ pair variances on $M Z$ pair means (Jinks and Fulker, 1970). In the absence of GXE interaction, directional dominance is indicated by significant regression of $D Z$ pair variances on $D Z$ pair sums, or by significant coefficients of skewness evident in $D Z$ twins only (Martin et al., 1978). The probability of detecting dominance by fitting models to twin data is generally low, even when there are complete dominance and high heritability, unless it has a strong directional component (Martin et al., 1982). As a test for GxE interactions and directional dominance in our data, the absolute pair difference, which is proportional to the square root of the intrapair variance, was regressed onto pair sum, and onto the square of the pair sum. In case the relationship was not linear, square and logarithmic (log) transformations of the data were also tested for significant regression. Coefficients of skewness were calculated and compared between $M Z$ and $D Z$ twin pairs.

Structural modeling was then implemented, with the program Mx (Neale, 1994) used to account for genetic and environmental covariation between the incisor crown dimensions. Implicit in our model-fitting procedure were all the usual assumptions of the twin method-that mating is random, that trait-related shared environmental influences on $M Z$ and $D Z$ twins are equal, and that there is no GxE interaction or gene-environment covariation (Jinks and Fulker, 1970). Fig. 1 shows four of the influences which can be modeled (A, C, D. 
Table 1. Percent measurements present, and percent substituted from each source

\begin{tabular}{lrrrr}
\hline Tooth & U1 & U2 & L1 & L2 \\
\hline Present & 96.6 & 93.8 & 98.6 & 98.0 \\
Other Side & 1.9 & 1.6 & 1.1 & 1.3 \\
Other Twin & 1.3 & 2.0 & 0.2 & 0.6 \\
Mean & 0.2 & 2.6 & 0.2 & 0.2 \\
Total & 3.4 & 6.2 & 1.4 & 2.0 \\
\hline
\end{tabular}

2 $U=$ upper, $L=$ lower, 1 = central incisor, 2 = lateral incisor.

and $E$ ) for a pair of twins. The correlations of $A 1$ with $A 2$ and D1 with D2 are fixed according to genetic theory. Additive genetic variation causes $D Z$ correlations to be about half the $M Z$ correlations. Dominance tends to decrease the $\mathrm{DZ}$ correlation to below half the $M Z$ value, and common environment increases it above half the $M Z$ value. By definition, unique environmental influences are uncorrelated, and shared environmental influences are perfectly correlated. Since fitting models with four parameters to data from a classical twin study $(M Z$ and $D Z$ twins reared together) results in an underidentified model, subsets of three or fewer parameters are chosen. The choice is made simpler by negative confounding of genetic dominance with common environmental influences (Grayson, 1989; Hewitt, 1989), so that a twin model may not contain both $D$ and $C$.

Variable-length files of raw data were set up as described in Neale (1994) and utilized directly for the univariate analyses. For the multivariate analyses, the input data took the form of variance-covariance matrices, generated for each of the five twin sex-zygosity groups, by means of the preprocessor, PRELIS (Jöreskog and Sörbom, 1986). List-wise deletion of twin pairs with one or more missing values, necessary to produce positive-definite variance-covariance matrices, would have resulted in the loss of up to $26 \%$ of the data. Since the proportion of the total data set missing was reasonably small, ranging from $1.4 \%$ for the lower lateral incisor to $6.2 \%$ for the upper lateral incisor (Table 1), an imputation procedure was applied following a substitution hierarchy. Values were substituted from the antimeric tooth when present. If the antimere was absent, the value from the co-twin was used in same-sexed twin pairs. Where this was also missing, or the twins were opposite-sexed, the sex-specific mean was used. To reduce the impact of the imputations, we removed twin pairs with fewer than 60 of the 112 values present (56 values in each twin), leaving $78 \mathrm{MZ}$ female, $61 \mathrm{MZ}$ male, $44 \mathrm{DZ}$ female, $41 \mathrm{DZ}$ male, and $48 \mathrm{DZ}$ male-female twin pairs. The age range for the 272 twin pairs was consequently reduced to between nine and 46 vears.

We began by analyzing each variable separately, fitting a path coefficient model with unique environmental influences only ( $E$ model). Where this failed, the model was extended to include additive genetic variation ( $A E$ model), or shared environmental variation (CE model). Finally, ACE and ADE models were fitted, where $D$ (non-additive genetic variation) incorporates both dominance and epistatic interaction variance, which cannot be separated when only $M Z$ and $D Z$ twins are used (Mather, 1974). Path coefficients (a, d, c, e) were estimated, and $\chi^{2}$ values for goodness-of-fit of the models were calculated.

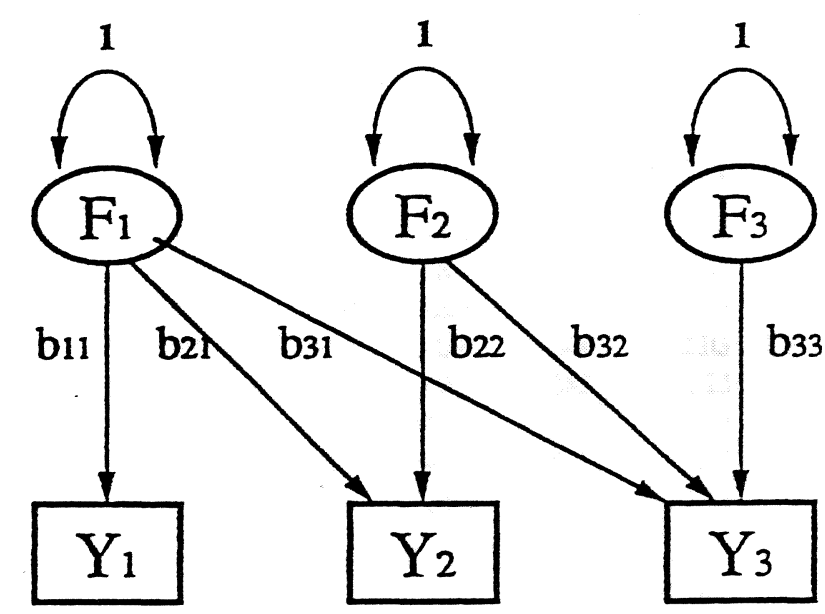

Figure 2. Path diagram depicting a Cholesky decomposition model with three measured variables $\left(Y_{1}\right.$ to $Y 3$ ) being explained by three latent factors ( $\mathrm{Fl}$ to $\mathrm{F} 3$ ). The double-headed arrows indicate the variance of the latent factors (from Neale and Cardon, 1992).

Akaike's Information Criterion (AIC $=\chi^{2}$ minus two times the degrees of freedom) was used to indicate the parsimony of each model (Akaike, 1987). The smaller or more negative the AIC, the better the parsimony and fit of a model. The general approach is that of accepting a more complex model only when a simpler one has failed. In addition, comparisons of the $x^{2}$ and AIC values between complex and simpler models may indicate significance of the various components. Various hypotheses can be tested by setting different combinations of paths to zero, and examining the difference between the resulting goodness-of-fit $x^{2}$ and AIC values. We estimated heritability $\left(h^{2}\right)$ from the ratio of genetic variation to total phenotypic variation, using the parameter estimates from the model with the best fit.

The most parsimonious mudel for each tooth was applied to four subsets of the data base, namely, female same-sexed twin pairs, male same-sexed twin pairs, all four same-sexed twin groups, and the five sex-zygosity groups. We evaluated heterogeneity of causes of variation between the sexes by adding the $\chi^{2}$ values for the fits of the model to male and female groups separately, and then subtracting this sum from the $\chi^{2}$ generated by fitting the model jointly to the four groups.

The multivariate analysis was conducted for each gender separately and was comprised of three main steps, each of which utilized the best model from the previous step. In the first stage, Cholesky decomposition models were applied to all eight variables. These models estimate all possible paths of covariation in an attempt to account for as much variation as possible, having as many factors as there are variables and as many loadings as there are observed correlations. The path diagram of a Cholesky decomposition of three variables ( $Y 1, Y 2$, $\mathrm{Y} 3$ ) into three factors (F1, F2, F3) is shown in Fig. 2. The first factor (F1) loads on all the variables, the second (F2) loads on all but the first variable, the third (F3) loads on all but the first and second variables, and so on (Neale and Cardon, 1992). The Cholesky model is a unique factorization of the covariance structure. It therefore provides a limiting test of how w'ell any model with $A, E, C$, or D factors will fit. Simpler models will 
Table 2 Descriptive statistics for the mesiodistal dimension (mm) of permanent incisors in each sex

\begin{tabular}{lccccccc}
\hline & \multicolumn{3}{c}{ Males } & & \multicolumn{3}{c}{ Females } \\
\cline { 2 - 5 } \cline { 6 - 8 } Tooth $^{\mathbf{a}}$ & $\mathrm{N}^{\mathrm{b}}$ & Mean & SD & & $\mathrm{N}$ & Mean & SD \\
\hline UR2 & 237 & 6.87 & 0.56 & & 269 & 6.62 & 0.53 \\
UR1 & 240 & 8.82 & 0.55 & & 288 & 8.52 & 0.56 \\
UL1 & 241 & 8.82 & 0.56 & & 282 & 8.53 & 0.54 \\
UL2 & 238 & 6.87 & 0.59 & & 277 & 6.61 & 0.57 \\
LR2 & 248 & 6.05 & 0.41 & & 289 & 5.84 & 0.41 \\
LR1 & 246 & 5.44 & 0.37 & & 286 & 5.26 & 0.38 \\
LL1 & 249 & 5.44 & 0.35 & & 285 & 5.26 & 0.37 \\
LL2 & 246 & 6.05 & 0.41 & & 289 & 5.83 & 0.40 \\
\hline
\end{tabular}

- $U=$ upper, $L=$ lower, $R=$ right, $L=$ left, 1 = central incisor, 2 = lateral incisor.

b $\mathrm{N}=$ sample size; $\mathrm{SD}=$ standard deviation.

c Male and female distributions significantly different $(p<0.001)$ for all variables.

display a worse fit than this by the $x^{2}$ criterion, but are preferred if more parsimonious (as estimated by AIC) or more appropriate on theoretical grounds. The first model again consisted of an E matrix alone, followed by $A E, C E, A C E$, and ADE models.

In the second stage, principles of parsimony and biological theory were used to test models involving combinations of factors loading on all eight incisors ("general" factors) and factors loading on one or more pairs of incisors ("group" factors). Once a favorable genetic model was determined, the third step involved the same approach to elucidate the structure of the individual environmental covariation. Finally, as in the univariate analysis, $x^{2}$ tests of heterogeneity between male and female data were applied to the most parsimonious models, and estimates of heritability were obtained.

\section{Results}

\section{Measurement reliability}

The mean squared differences between the sets of measurements obtained by the two investigators were small, with no value exceeding $0.01 \mathrm{~mm}$. The technical error of measurement, or Dahlberg statistic (Dahlberg, 1940), averaged $0.06 \mathrm{~mm}$, with a range of from 0.04 to $0.07 \mathrm{~mm}$. The reliability of the measurement technique was estimated as the ratio of true to observed variance, where the true variance was calculated as the observed minus the error variance. For our test-retest data, the estimated (interobserver) reliability of measuring dental casts ranged from 0.96 to 0.99 , with an average value of 0.98 .

\section{Descriptive statistics}

The mean values and standard deviations for each crown dimension in males and females are listed in Table 2. Student's $t$ tests revealed significant differences in mean values between the sexes for all eight incisors ( $p<0.001$ ), with males having larger teeth than females. Variance ratio tests revealed no significant differences in variances between the sexes ( $p>0.05$ ). There was no evidence of a relationship between mean and variance from either variance ratio tests
Table 3. Correlation coefficients (x100) of incisor mesiodistal dimensions for each sex

\begin{tabular}{lcccccccc}
\hline & & & & \multicolumn{4}{c}{ Females $(\mathbf{N}=278$ to 308) } \\
& UR2 & UR1 & ULI & UL2 & LR2 & LR1 & LL1 & LL2 \\
\hline UR2 & - & 66 & 62 & $90^{2}$ & 54 & 52 & 52 & 54 \\
UR1 & 56 & - & 94 & 61 & 69 & 71 & 74 & 70 \\
UL1 & 55 & 91 & - & 62 & 69 & 70 & 72 & 71 \\
UL2 & 88 & 56 & -56 & - & 51 & 49 & 49 & 53 \\
LR2 & 53 & 66 & 67 & 53 & - & 73 & 75 & 88 \\
LR1 & 46 & 63 & 63 & 46 & 71 & - & 89 & 73 \\
LL1 & 45 & 60 & 61 & 45 & 65 & 88 & - & 73 \\
LL2 & 46 & 62 & 64 & 49 & 85 & 68 & 63 & - \\
\hline
\end{tabular}

Males (N $=254$ to 273$)$

- Values in bold type represent correlations between antimeric tooth pairs.

All values are significant $(p<0.001)$. $N$ values vary due to different numbers of missing values.

or regression analysis of pair variances on pair sums. Further $t$ tests revealed no significant differences in average incisor crown size between first- and second-born twins or between the zygosities in either sex $(p>0.05)$.

Correlation coefficients for pairs of tooth dimensions within each sex are given in Table 3. All correlations were significant $(p<0.001)$. The strongest correlations were between antimeric teeth, with values ranging from 0.85 to 0.94 . Table 4 displays the correlation coefficients between cotwins for each of the sex/zygosity groups. Values for $M Z$ twins ranged from 0.79 to 0.90 ( $p<0.001$ ), those for $D Z$ same-sexed twins from 0.33 to 0.76 ( $p<0.05$ ), and those for $D Z$ opposite-sexed twins from 0.10 to 0.43 (most $p<0.05$ ). The standard errors of the between-twin correlations for the $D Z$ twin groups (from 0.11 to 0.15 ) were approximately double those for the $\mathrm{MZ}$ twins (from 0.05 to 0.07 ).

Testing for genotype by environment interaction and directional dominance

For the raw data, regressions of absolute pair difference on pair sum and pair sum squared did not suggest $G \times E$ interaction or directional dominance. Only two of the $\mathbf{4 0}$ regressions (eight teeth by five twin groups) of absolute pair difference on pair sum were significant $(p<0.05)$, these being for the lower left lateral incisor in $\mathrm{MZ}$ males and the lower left central incisor in $\mathrm{DZ}$ females. The same variables provided significant results for the regression on pair sum squared, as did the lower right lateral incisor in $M Z$ males. The square and $\log$ transformations of the data did not improve linearity. Above all, there was no evidence of stronger relationship between these variables in $D Z$ compared with $M Z$ twins. These tests provide little evidence of dominance in the data.

\section{Univariate analyses}

A model with only a unique environmental factor (E) was rejected ( $p<0.001$ ) for all groups and all variables. The AE model was adequate for all variables except for the upper right central incisor in the "Male" group and in the "All" group. Table 5 a shows the squared standardized parameter estimates for the AE models fitted to each incisor crown 
Table 6. Goodness-of-fit statistics for the multivariate analysis in female and male twins

\begin{tabular}{|c|c|c|c|c|c|c|c|c|}
\hline \multirow[b]{2}{*}{ No. } & \multirow[b]{2}{*}{ Model } & \multirow[b]{2}{*}{$d P$} & \multicolumn{3}{|c|}{ Females } & \multirow[b]{2}{*}{$x^{2}$} & \multicolumn{2}{|c|}{ Males } \\
\hline & & & $x^{2}$ & Prob & $\mathrm{AIC}^{\mathrm{b}}$ & & Prob & AIC \\
\hline & STEP 1: Cholesky models & & & & & & & \\
\hline 1 & $\mathrm{ACE}$ & 164 & 236.74 & $<0.001$ & -91.26 & 244.22 & $<0.001$ & -83.78 \\
\hline 2 & $A D E$ & 164 & 236.65 & $<0.001$ & -91.35 & 256.69 & $<0.001$ & -71.31 \\
\hline 3 & $\mathbf{A E}$ & 200 & 243.86 & 0.019 & -156.14 & 265.94 & 0.001 & -134.06 \\
\hline 4 & $\mathrm{CE}$ & 200 & 360.71 & $<0.001$ & -39.29 & 346.89 & $<0.001$ & -53.11 \\
\hline \multirow[t]{2}{*}{5} & $\mathbf{E}$ & 236 & 644.34 & $<0.001$ & 172.34 & 647.98 & $<0.001$ & 175.98 \\
\hline & STEP 2: Model 3, vary A & & & & & & & \\
\hline 6 & Cholesky, 5 factors & 206 & 243.86 & 0.036 & -168.14 & 271.11 & 0.002 & -140.89 \\
\hline 7 & Cholesky, 4 factors & 210 & 245.65 & 0.046 & -174.35 & 279.15 & 0.001 & -140.85 \\
\hline \multirow[t]{2}{*}{8} & General, Upper, Lower & & & & & & & \\
\hline & Lateral, Central & 212 & 244.63 & 0.062 & -179.37 & 276.19 & 0.002 & -147.81 \\
\hline 9 & General + 4 group factors & 220 & 252.43 & 0.066 & -187.57 & 290.00 & 0.001 & -150.00 \\
\hline 10 & General factor only & 228 & 445.76 & $<0.001$ & -10.24 & 459.10 & $<0.001$ & 3.10 \\
\hline 11 & Group factors only & 228 & 435.64 & $<0.001$ & -20.36 & 463.06 & $<0.001$ & 7.06 \\
\hline 12 & - With symmetry in: & & & & & & & \\
\hline 13 & - General factor & 224 & 254.20 & 0.081 & -193.80 & 311.46 & $<0.001$ & -136.54 \\
\hline 14 & - Group factors & 224 & 257.11 & 0.064 & -190.89 & 299.15 & $<0.001$ & -148.85 \\
\hline \multirow[t]{2}{*}{15} & - All & 228 & 258.96 & 0.078 & -197.04 & 317.32 & $<0.001$ & -138.68 \\
\hline & STEP 3: Model 15, vary E & & & & & & & \\
\hline 16 & General +8 specific factors ${ }^{d}$ & 248 & 327.27 & 0.001 & -168.73 & 343.74 & $<0.001$ & -152.26 \\
\hline 17 & -Specific factors only & 256 & 400.90 & $<0.001$ & -111.10 & 367.28 & $<0.001$ & -144.72 \\
\hline 18 & - Model 16, with symmetry & 256 & 338.34 & $<0.001$ & -173.66 & 351.99 & $<0.001$ & -160.01 \\
\hline
\end{tabular}

$\mathrm{df}=$ degrees of freedom.

- Akaike's Information Criterion (AIC) $=\chi^{2}-2 \mathrm{df}$.

- Group factors represent the four antimeric tooth pairs.

d Specific factors represent each of the eight incisors. The best model in each step is highlighted in bold.

constraints were applied to the additive genetic factors (Model 15). The model for male data was improved by symmetry constraints on all but the general genetic factor, which was better left unconstrained (Model 14). The $x^{2}$ value increased by 18.2 ( $4 \mathrm{df} ; \mathrm{p}<0.001$ ) when the general genetic factor was constrained (Model 15). The third step further investigated the environmental covariation, using the best-fitting model for the additive genetic covariation (Model 15). The factor loadings from the Cholesky decomposition of the unique environmental covariation are listed in Tables $7 \mathrm{a}$ and $7 \mathrm{~b}$. The largest factor loadings were on the diagonal of the matrix, indicating that the effects of environment on each tooth separately were greater than those on any groupings of the teeth. The second highest loadings involved antimeric teeth. Exploration of the unique environmental covariation thus began with models comprising a general factor affecting all eight incisors and eight specific factors, one for each tooth. When these submodels were applied for the unique environmental covariation, the shift from Cholesky decomposition to one general and eight specific factors improved the fit for the males, but worsened it for the females, with a difference in $\chi^{2}$ of 68.3 (20 df; $p<0.001$ ). As with the additive genetic variation, the differences in $\chi^{2}$ values between models indicated that the environmental covariation contained both group and specific factors. Comparison of models 16 and 17 yielded $x^{2}=73.6$ for females and $x^{2}=23.5$ for males ( $8 \mathrm{df} ; \mathrm{p}<0.01)$. The model with a general factor alone was so unlikely, it resulted in a nonsensical $\chi^{2}$ and probability level. Symmetry constraints on all factors improved the fit for both sexes (Model 18). The path diagram for this model is depicted in Fig. 3. The factor loadings of additive genetic and individual environmental components are summarized in Fig. 4. Heritability estimates $\left(h^{2}\right)$ ranged from 0.81 to 0.91 in females, and from 0.84 to 0.89 in males (Fig. 5).

Model 18 was used to test the data for heterogeneity of fit between sexes. This model produced a goodness-of-fit of $x^{2}=$ 338.3 for females, $x^{2}=352.0$ for males ( $256 \mathrm{df} ; \mathrm{p}<0.001$ ), and $x^{2}=$ 724.1 ( $528 \mathrm{df} ; \mathrm{p}<0.01$ ) when fitted to all four same-sexed matrices. Subtracting the sum of the $\chi^{2}$ values for the sexes considered separately from the third (joint) fit gave a heterogeneity Chi-square of $\chi^{2}=33.8$ ( $16 \mathrm{df} ; \mathrm{p}<0.01$ ), indicating significant heterogeneity between female and male twins.

\section{Discussion}

\section{Descriptive statistics}

The only significant difference in the distributions of male and female data involved the means, with males having larger incisors than females. The correlation analysis provided evidence of common environmental influences on the upper central incisors in males, since $\mathrm{DZ}$ correlations were almost as high as those for $M Z$ twins. In fact, the $D Z$ correlations were greater than half the $M Z$ correlations for all eight variables in the males, especially for all central incisors and for the lower central incisors in the females. 
Table 4. Between-twin correlation coefficients $(x 100)$ for incisor mesiodistal dimensions, within each of the five sex/zygosity groups

\begin{tabular}{|c|c|c|c|c|c|c|c|c|c|}
\hline Twin Group & $N($ pairs)d & UR2 & UR1 & UL1 & UL2 & LR2 & LR1 & LLI & LL2 \\
\hline \multicolumn{10}{|l|}{ MZ Twins } \\
\hline $\mathbf{F F}$ & $72-81$ & $87(06)^{b}$ & $90(05)$ & $89(05)$ & $85(06)$ & $84(06)$ & $79(07)$ & $87(06)$ & $82(07)$ \\
\hline $\mathrm{MM}$ & $63-67$ & $87(06)$ & $82(07)$ & $86(07)$ & $89(06)$ & $84(07)$ & $86(06)$ & $87(06)$ & $85(07)$ \\
\hline \multicolumn{10}{|l|}{ DZ Twins } \\
\hline FF & $42-46$ & $4.5(14)$ & $40(14)$ & $41(14)$ & $41(14)$ & $39(14)$ & $52(13)$ & $50(13)$ & $33(14)$ \\
\hline MF & $45-55$ & $10(15)^{x}$ & $33(13)$ & $29(13)$ & $25(15)^{c}$ & $43(13)$ & $42(13)$ & $22(14)^{c}$ & $39(13)$ \\
\hline
\end{tabular}

- $F=$ female, $M=$ male. All other abbreviations as in Table 2

b Numbers in parentheses represent standard errors associated with the correlation coefficients.

c Correlation not significantly different from zero ( $p>0.05$ ).

$\mathrm{N}$ values vary due to different numbers of missing values.

dimension separately, within each of the data subsets. Equivalent parameter estimates for the ACE models fitted to

Table 5a. Squared standardized parameter estimates (x100) for univariate AE models applied to incisor mesiodistal dimensions

\begin{tabular}{|c|c|c|c|c|}
\hline Tooth & Data Subset & $a^{2 a}$ & $e^{2}$ & $x^{2 b}$ \\
\hline \multirow[t]{4}{*}{ UR2 } & Females & 86 & 14 & 7.10 \\
\hline & Males & 85 & 15 & 3.82 \\
\hline & Same Sex & 85 & 15 & 12.59 \\
\hline & All & 86 & 14 & 25.97 \\
\hline \multirow[t]{4}{*}{ UR1 } & Females & 90 & 10 & 3.24 \\
\hline & Males & 84 & 16 & $19.44^{\mathrm{C}}$ \\
\hline & Same Sex & 87 & 13 & $25.15^{d}$ \\
\hline & All & 88 & 12 & $33.65^{e}$ \\
\hline \multirow[t]{4}{*}{ UL1 } & Females & 89 & 11 & 2.28 \\
\hline & Males & 89 & 11 & $1244^{\mathrm{d}}$ \\
\hline & Same Sex & 89 & 11 & 15.57 \\
\hline & All & 90 & 10 & 21.35 \\
\hline \multirow[t]{4}{*}{ UL2 } & Females & 85 & 15 & 2.16 \\
\hline & Males & 88 & 12 & 6.92 \\
\hline & Same Sex & 87 & 13 & 10.14 \\
\hline & All & 87 & 13 & 17.80 \\
\hline \multirow[t]{4}{*}{ LR2 } & Females & 82 & 18 & 9.22 \\
\hline & Males & 84 & 16 & 6.63 \\
\hline & Same Sex & 83 & 17 & 16.28 \\
\hline & All & 83 & 17 & 19.62 \\
\hline \multirow[t]{4}{*}{ LR1 } & Females & 78 & 22 & 4.64 \\
\hline & Males & 86 & 14 & 3.37 \\
\hline & Same Sex & 82 & 18 & 12.64 \\
\hline & All & 82 & 18 & 19.94 \\
\hline \multirow[t]{4}{*}{ LL1 } & Females & 85 & 15 & 4.30 \\
\hline & Males & 89 & 11 & 2.08 \\
\hline & Same Sex & 86 & 14 & 10.23 \\
\hline & All & 88 & 12 & 21.63 \\
\hline \multirow[t]{4}{*}{ LL2 } & Females & 82 & 18 & 4.91 \\
\hline & Males & 86 & 14 & 3.26 \\
\hline & Same Sex & 84 & 16 & 8.76 \\
\hline & All & 85 & 15 & 13.57 \\
\hline
\end{tabular}

$\mathrm{a}^{2}=$ additive genetic variation, $\mathrm{e}^{2}=$ unique environmental variation.

b Degrees of freedom for $x^{2}$ are 7 for females and males, 16 for "Same-Sex", and 21 for "All" twins for the AE model.

c $\mathrm{p}<0.01 ;{ }^{d} 0.05<\mathrm{p}<0.10 ;{ }^{e} \mathrm{p}<0.05$. the upper central incisors are given in Table 5b. The ACE model displayed a significantly better fit than the AE model for the upper right central incisor of males, with a difference between the two $x^{2}$ values of 7.94 [1 degree of freedom (d); $p<$ 0.005 ]. Low probabilities (from 0.05 to 0.10 ) were also obtained for the upper left central incisor, $A E$ and $A C E$ models, in the males. Significant heterogeneity in fit of the ACE model occurred between males and females for the upper right central incisor $(p<0.05)$, while there was no significant difference in fit for the upper left central incisor ( $p>0.95$ ).

Multivariate analyses

The results of the first stage of the multivariate analysis (Table 6) were consistent with those of the univariate analyses, in that the AE model displayed the best fit for each gender (Model 3). In the second stage, one of the best-fitting models for the additive genetic covariation (Model 9) was comprised of a general factor and four group factors, one each for the antimeric pairs of incisors. The general genetic factor alone was demonstrated to be insufficient, with the differences in $\chi^{2}$ values between Models 9 and 10 being 193.3 for females and 169.1 for males ( $8 \mathrm{df} ; \mathrm{p}<0.001$ ). The group factors $\mathrm{fc}$. antimeric tooth pairs were also insufficient on their own, with the differences in $x^{2}$ values between Models 9 and $1 \mathrm{i}$ being 183.2 for females and 173.1 for males $(8 \mathrm{df}, \mathrm{p}<$ $0.00 \%$. For the females, the fit improved when symmetry

Table 5b. Squared standardized parameter estimates $(x 100)$ for univariate ACE models applied to mesiodistal dimensions of the upper central incisors

\begin{tabular}{lllrcc}
\hline Tooth & Data Subset & $\mathrm{a}^{2}$ & $\mathrm{c}^{2 \mathrm{a}}$ & $\mathrm{e}^{2}$ & $\mathrm{x}^{2 \mathrm{~b}}$ \\
\hline UR1 & Females & 90 & 0 & 10 & 3.24 \\
& Males & 28 & 56 & 16 & $11.50^{\mathrm{c}}$ \\
& Same Sex & 65 & 22 & 13 & $23.16^{\mathrm{c}}$ \\
& All & 88 & 0 & 12 & $33.65^{\mathrm{c}}$ \\
UL1 & Females & 89 & 0 & 11 & 2.28 \\
& Males & 65 & 24 & 11 & $11.12^{\mathrm{c}}$ \\
& Same Sex & 80 & 9 & 11 & 15.23 \\
& All & 90 & 0 & 10 & 21.35 \\
\hline
\end{tabular}

$c^{2}=$ common environmental variation.

b Degrees of freedom for $\chi^{2}$ are 6 for Females and Males, 15 for "Same-Sex", and 20 for "All" turins for the ACE model.

c $0.05<p<0.10 ;{ }^{d} p<0.05$. 
Beyond this, the correlations suggested an influence of unique environment-since $M Z$ correlations were less than one-and additive genetic factors where $\mathrm{DZ}$ correlations were approximately half the $M Z$ values. The difference in standard errors of the correlations between $\mathrm{MZ}$ and $\mathrm{DZ}$ twins is partly due to the smaller sample sizes of $D Z$ compared with $M Z$ twins, and partly indicative of genetic infiuence on tooth size.

\section{Genetic and environmental components}

The main finding from both uni- and multivariate modelfitting was that variation in incisor crown size was explicable mostly by additive genetic and individual environmental variance, with no need for non-additive genetic or shared environmental variance. This supports the previous finding for the upper lateral incisor in Pima Indians (Potter et al., 1983), in which most of the genetic variation was additive, with unique environmental effects as well. An expected artifact of the imputation procedure used for the multivariate analyses was the spurious occurrence of common environment, caused by the substitution of sexspecific means or values from the co-twin. Since common environment was not a significant factor in these analyses, the imputation procedure did not appear to have any significant impact on our findings.

Our analyses revealed a general genetic factor (gene or group of genes) which influenced all of the incisors, in contrast to the evidence of independent genetic determination of maxillary and mandibular teeth advocated by Potter et al. (1976). However, the finding is consistent with studies of individuals with chromosomal abnormalities (e.g., Alvesalo et al., 1991), implying that human sex chromosomes influence the thickness of dental crowns, and also with recent molecular genetic investigations (Lau et al., 1989; Nakahori et al., 1991; Salido et al., 1992) showing that genes on the human sex chromosomes influence enamel formation. It has been hypothesized further that sequence differences between the genes on $X$ and $Y$ chromosomes contribute to the observed sexual dimorphism in tooth size (Lau et al., 1990; Fincham et al., 1991). In addition to a general genetic factor, there were additive genetic influences on antimeric pairs of teeth, and unique environmental factors operating on each soth, and on the eight incisors as a group. The slight iea. tion in goodness-of-fit to the female data which occurred when the model was changed from a Cholesky decomposition to one general and eight specific unique environmental factors was not considered biologically significant, but more a reflection of the power of the twin study to detect environmental influences, especially since the factor loadings displayed a pattern very similar to those in the males (Tables $7 a$ and $7 b$ ).

The only exception to the finding of additive genetic and individual environmental influences in our data involved the upper right central incisor in male twins, for which there was evidence of a common environmental effect. This is consistent with the relative magnitudes of the correlation coefficients in male $D Z$ and $M Z$ twins. The significant heterogeneity between females and males. for this tooth suggests that genetic and environmental factors differed significantly between the sexes. On re-checking the data for outliers, we noted a pair of male DZ twins with unusually

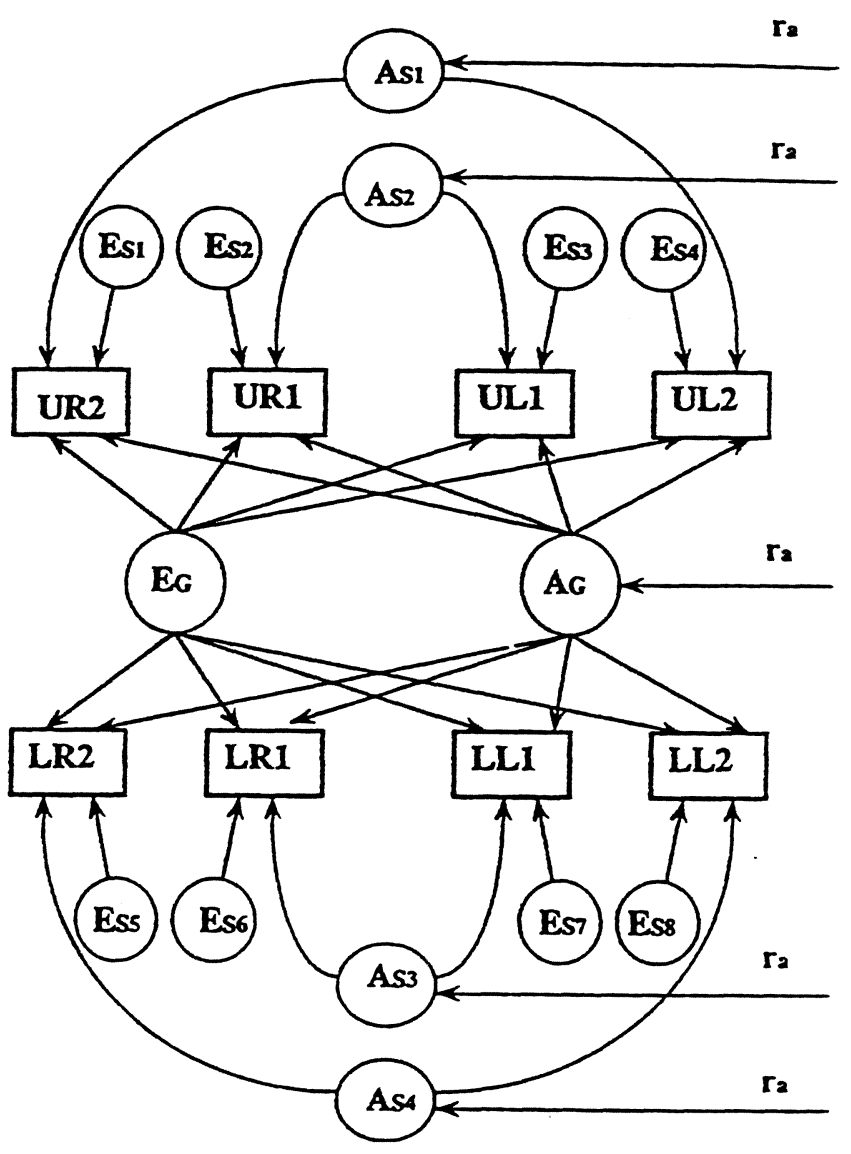

Figure 3. Path diagram for the best-fitting multivariate model (Model 18), illustrating the hypothesized covariance structure of the mesiodistal dimension for the eight incisor crowns in one twin. $A_{G}$ and $A_{S(1-1)}$ denote the general and group additive genetic factors, respectively, for twin 1, while $E_{C}$ and $E_{S(1-8)}$ denote general and specific unique environmental factors in twin 1 . The entire diagram should be duplicated for twin 2 , with the double-headed arrows indicating correlations $\left(r_{2}=1.0\right.$ for $M Z, 0.5$ for $D Z$ twins) between the twin members.

small teeth. When they were excluded from the analyses, the correlations decreased slightly, but the univariate and multivariate analyses and the $x^{2}$ test for heterogeneity in the multivariate analyses remained unchanged. It is therefore unlikely that outliers were responsible for the apparent effect of shared environment.

One potential source of shared environmental contribution to tooth size is the hormonal composition of the uterine environment. In humans, males have larger teeth on average than females. If androgens contribute to increased tooth size and are able to diffuse from one twin to the other, then we might predict an increased similarity in dental dimensions of male DZ twins. This would be reflected in statistical analyses as a common environmental effect in males. In other mammals, testosterone diffuses between fetuses through the amniotic membranes (Fels and Bosch, 1971), or via the maternal circulation (Meisel and Ward, 1981). Indirect evidence for hormonal exchange between human twins arises from a preliminary study of opposite-sexed twins, in which we noted a trend toward larger teeth in females with twin 

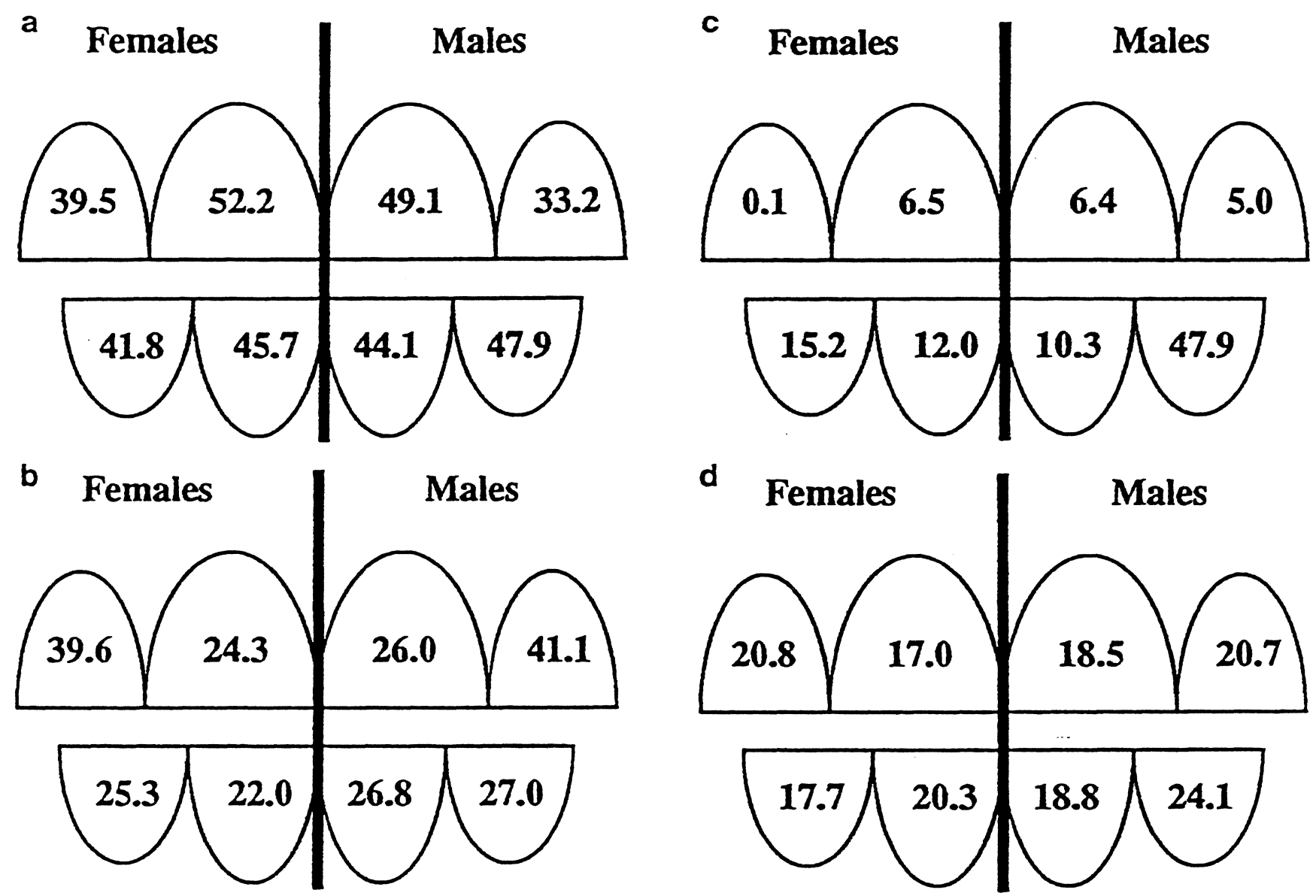

Figure 4. Standardized factor loadings (x100) from Model 18 in females (left) and maies (right). Only one side of the dentition is displayed, since loadings are identical on each side. (a) Loadings for the general additive genetic factor. (b) Loadings for the additive genetic factor for antimeric pairs of teeth. (c) Loadings for the general unique environmental factor. (d) Loadings for the unique environmental factor for individual teeth.

brothers, than in females with twin sisters (Dempsey et al., 1994), indicating a possible masculinizing effect on females. However, if this type of sibling interaction does occur, one would expect to find decreased variance among male $\mathrm{DZ}$ twins relative to male $M Z$ twins (Neale and Cardon, 1992). No such decrease in variance was found in our data.

The lack of evidence for non-additive genetic variance in our data does not necessarily mean that it does not exist. Since the preliminary regression analyses of pair sums on pair differences showed no evidence of directional dominance, there was less a priori chance of detecting non-additive genetic variation. Additionally, if common environmental influences do exist, they will inflate additive genetic variation and deflate non-additive genetic variation (Martin et al., 1978). Thus, the apparent absence of both non-additive genetic variation and shared environmental variation may be due to having insufficient power to detect them, a difficulty which further sampling may resolve. However, if these contributing factors did exist in our data, they are likely to have been small by comparison with the contributions of additive genetic and unique environmental factors. It is interesting to note that dominance was not a necessary componerit of the model developed by Potter et al. (1983), although statistical power was also a problem in their study.
The advantages of the modeling techniques applied in this analysis include greater ability to separate genetic from non-genetic effects, additive from non-additive genetic variations, and individual from familial environment. It was also possible to test the data for genotype-environment interactions, and to analyze the eight variables simultaneously. Most earlier studies applied heritability estimation procedures to univariate data. The estimates incorporated a number of inseparable genetic and environmental variance components, and/or assumed no genetic interaction (epistasis) or genotype-environment interaction (Bulmer, 1970; Smith, 1974; Mizoguchi, 1977).

\section{Symmetry}

The multivariate study indicated that all of the factors for both additive genetic and individual environmental variation operated similarly on antimeric pairs of teeth. The conclusion that antimeric teeth shared the same genetic determinants is consistent with other accounts (e.g., Potter et al., 1976). In a study of dental crown traits in Mexican Indians and Afro-Belizeans, Baume and Crawford (1980) concluded that common genetic factors were likely to influence characters on both sides of the dental arch equally. Asymmetry was proposed to occur through local 
Table 72. Estimated factor loadings for the Cholesky decomposition of unique environmental variance in females

\begin{tabular}{|c|c|c|c|c|c|c|c|c|}
\hline Females & UR2 & UR1 & ULI & UL2 & LR2 & LRI & LL1 & LL2 \\
\hline UR2 & $0.1837^{a}$ & & & & & & & \\
\hline UR1 & $0.0608^{\mathrm{a}}$ & 0.1703 & & & & & & \\
\hline ULI & 0.0071 & 0.0866 & 0.1553 & & & & & \\
\hline UL2 & 0.0698 & 0.0242 & 0.0113 & 0.2068 & & & & \\
\hline LR2 & -0.0104 & 0.0384 & 0.0391 & -0.0032 & 0.1585 & & & \\
\hline LR1 & -0.0046 & 0.0031 & 0.0097 & 0.0134 & 0.0458 & 0.1530 & & \\
\hline LLI & -0.0080 & 0.0225 & -0.0074 & -0.0026 & 0.0528 & 0.0364 & 0.1215 & \\
\hline LI2 & -0.0264 & 0.0164 & 0.0181 & -0.0046 & 0.0781 & 0.0098 & 0.0063 & 0.1482 \\
\hline
\end{tabular}

Factor loadings in bold are $>0.1$; those in bold italics lie between 0.05 and 0.1 .

Table 7b. Estimated factor loadings for the Cholesky decomposition of unique environmental variance in males

\begin{tabular}{|c|c|c|c|c|c|c|c|c|}
\hline Males & UR2 & UR1 & ULI & UL2 & LR2 & LR1 & LLI & LL2 \\
\hline UR2 & 0.2183 & & & & & & & \\
\hline URI & -0.0395 & 0.1828 & & & & & & \\
\hline UL1 & -0.0157 & 0.0546 & 0.1725 & & & & & \\
\hline UL2 & 0.0713 & 0.0015 & 0.0080 & 0.1869 & & & & \\
\hline LR2 & 0.0064 & 0.0111 & -0.0171 & -0.0104 & 0.1620 & & & \\
\hline LR1 & 0.0190 & 0.0072 & -0.0527 & 0.0110 & 0.0142 & 0.1216 & & \\
\hline LLI & 0.0192 & 0.0056 & -0.0122 & -0.0026 & 0.0095 & 0.0531 & 0.1001 & \\
\hline LL2 & 0.0077 & 0.0514 & 0.0291 & 0.0092 & 0.0361 & 0.0059 & -0.0021 & 0.1407 \\
\hline
\end{tabular}

environmental conditions within the jaw or by more general intra-uterine developmental effects. Our model for unique environmental factors, like that of Potter et al. (1976), comprised independent environmental influences on right and left sides, although constraining antimeric loadings to be equal for unique environmental factors improved the fit of the model, indicating that the influences tended to have the same degree of impact on both sides.

\section{Heritability}

Our estimates of heritability, averaging $86 \%$, were reasonably high when compared with estimates of $35 \%$ (Potter et al., 1983), 54\% (Alvesalo and Tigerstedt, 1974), 60\% (Goose, 1971), 64\% (Townsend and Brown, 1978a,b), and up to $72 \%$ (Rebich and Markovic, 1976) in other studies of human tooth size. They were more compatible with Garn's (1977) estimate of up to $90 \%$. The variation in estimates of heritability among these studies reflects the different statistical approaches used, and probably also the different populations from which samples were drawn, since there may have been greater environmental effects within some than others. As predicted in Butler's field theory, the heritability estimates were slightly higher for both upper and lower central incisors than for the lateral incisors, although the differences were very small (3\% for upper and $1 \%$ for lower incisors). Expansion of our analyses to the rest of the dentition may shed more light on the theory, but at this stage there is little evidence of differential heritability with position in the incisors.

\section{Acknowledgments}

The South Australian Twin Study is supported by a grant from the National Health and Medical Research Council (NH \& MRC) of Australia, and by grants from the University of Adelaide. The assistance of the NH \& MRC Twin Registry is greatly appreciated. The twins and their families are also thanked for their enthusiastic participation in the study. Professor Tasman Brown, Drs. Brian Penhall, Lindsay Richards, John Kaidonis, and Jim Rogers, Mrs. Sandy Pinkerton, and Mrs. Wendy Schwerdt assisted with data collection. George Travan and John Pearson provided muchneeded computer support. Greg Johnston made helpful comments on the manuscript. We are also grateful to the staff of the Institute of Medical and Veterinary Science and Dr. John Mulley of the Adelaide Medical Centre for Women and Children, for their assistance in determining twin zygosities.

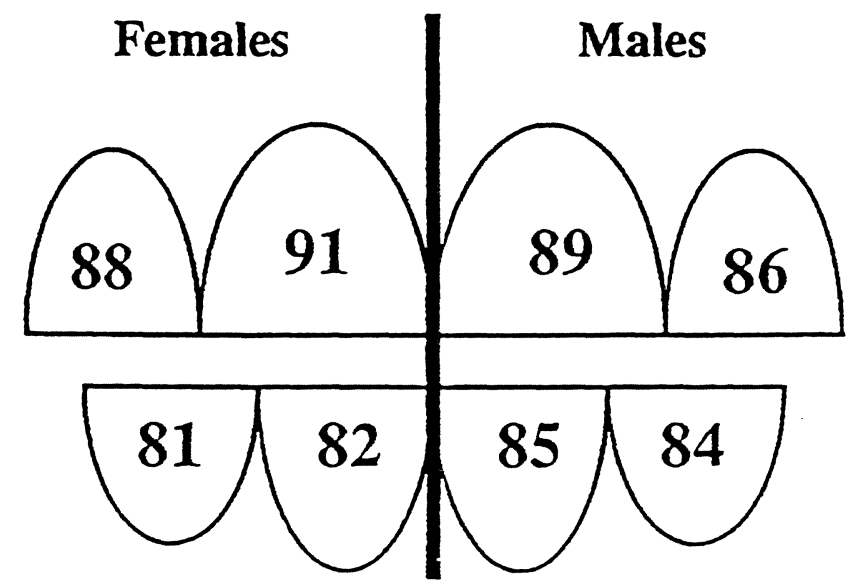

Figure 5. Estimated heritabilities $(x 100)$ for the mesiodista dimension of the permanent incisors in males and females. 
A preliminary report was presented at the Australasian Society for Human Biology Conference, 1993, and has been published as "Proceedings" of the conference.

\section{References}

Akaike H (1987). Factor analysis and AIC Psychometrika 52:317-332

Alvesalo L, Tigerstedt PMA (1974). Heritabilities of human tooth dimensions. Hereditas 77:311-318.

Alvesalo L, Tammisalo E, Townsend G (1991). Upper central incisor and canine tooth crown size in $47, X X Y$ males. J Dent Res 70:1057-1060.

Baume RM, Crawford MH (1980). Discrete dental trait asymmetry in Mexican and Belizean groups. Am J Phys Anthropol 52:315-321.

Bulmer MG (1970). The biology of twinning in man. Oxford: Clarendon Press.

Butler PM (1939). Studies of mammalian dentition. Differentiation of the post canine dentition. Proc Zool Soc Lond, Ser B 109:1-36.

Dahlberg AA (1945). The changing dentition of man. J Am Dent Assoc 32:676-690.

Dahlberg G (1940). Statistical methods for medical and biological students. London: George Allen and Unwin.

Dempsey PJ, Townsend GC, Richards LC (1994). Possible hormonal effects on tooth size in male-female twin pairs (abstract). J Dent Res 73:739.

Fels E, Bosch LR (1971). Effect of prenatal administration of testosterone on ovarian function in rats. Am / Obstet Gynecol 111:964-969.

Fincham AG, Bessem CC, Lau EC, Pavlova Z, Schuler C, Slavkin HC, et al. (1991). Human developing enamel proteins exhibit a sex-linked dimorphism. Calcif Tissue Int 48:288-290.

Garn SM (1977). Genetics of dental development. In: The biology of occlusal development. McNamara JA, editor. Ann Arbor, MI: University of Michigan, pp. 61-88.

Goose DH (1971). The inheritance of tooth size in British families. In Dental morphology and evolution. Dahlberg AA, editor. Craniofacial Growth Series. Ann Arbor Center for Human Growth and Development. Chicago: University of Chicago Press, pp. 144-149.

Grayson DA (1989). Twins reared together: minimizing shared environmental effects. Behav Genet 19:593-604.

Heath AC, Neale MC, Hewitt JK, Eaves LJ, Fulker DW (1989). Testing structural equation models for twin data using LISREL. Bchav Genet 19:9-35.

Hewitt JK (1989). Of biases and more in the study of twins reared together: a reply to Grayson. Behav Genet 19:605-608.

Jinks JL, Fulker DW (1970). Comparison of the biometrical genetical, MAVA and classical approaches to the analysis of human behaviour. Psychol Bull 73:311-349.

Jöreskog KG (1973). Analysis of covariance structures. In: Multivariate analysis III. Krishnaiah PR, editor. New York: Academic Press.

Jöreskog KG, Sörbom D (1986). PRELIS: A program for multivariate data screening and data summarization. A preprocessor for LISREL. Michigan: Scientific Software, Inc.

Kieser JA (1990). Human adult odontometrics: The study of variation in adult tooth size. Cambridge: Cambridge University Press.

Lau EC, Mohandas TK, Shapiro LJ, Slavkin HC, Snead ML (1989). Human and mouse amelogenin gene loci are on the sex chromosomes. Genomics 4:162-168.

Lau EC, Slavkin HC, Snead ML (1990). Analysis of human enamel genes: insights into genetic disorders of enamel. Cleft Palate / 27:121-130.

Lauweryns I, Carels C, Vlietinck R (1993). The use of twins in dentofacial genetic research. Am J Orthod Dentofac Orthop 103:33-38.

Lundström A (1948). Tooth size and occlusion in twins. New York: S. Karger.

Martin NG, Eaves LJ (1977). The genetical analysis of covariance structure. Heredity 38:79-95.

Martin NG, Eaves LJ, Kearsey MJ, Davies P (1978). The power of the classical twin study. Heredity 40:97-116.

Martin NG, Loesch DZ, Jardine R, Berry HS (1982). Evidence for directional non-additivity in the genetics of finger ridge counts. Ann Hum Biol 9:253-263.

Mather K (1974). Non-allelic interactions in continuous variation of randomly breeding populations. Heredity 32:414-419.

Meisel RL, Ward IL (1981). Fetal female rats are masculinized by male littermates located caudally in the uterus. Science 213:239-241.

Mizoguchi Y (1977). Genetic variability of permanent tooth crowns as ascertained from twin data. I Anthropol Soc Nippon 85:301-309.

Moorrees CFA, Thomsen SO, Jensen E, Yen PKJ (1957). Mesiodistal crown diameters of the deciduous and permanent teeth in individuals. I Dent Res 36:39-47.

Nakahori Y, Takenaka O, Nakagome Y (1991). A human X-Y homologous region encodes "amelogenin". Genomics 9:264-269.

Neale MC (1994). Mx Statistical modeling. 2nd ed. Richmond, VA: Department of Psychiatry, Box 710, MCV, Richmond, VA 23298, USA.

Neale MC, Cardon LR (1992). Methodology for genetic studies of twins and families. Dordrecht, The Netherlands: Kluwer Academic Publishers.

Potter RH, Nance WE, Yu P, Davis WB (1976). A twin study of dental dimension. II. Independent genetic determinants. Am J Phys Anthropol 44:397-412.

Potter RHY, Rice JP, Dahlberg AA, Dahlberg T (1983). Dental size traits within families: path analysis for first molar and lateral incisor. Am J Phys Anthropol 61:283-289.

Rebich T, Markovic M (1976). Inheritance of tooth dimension. A quantitative genetic twin study. 52nd Congress, June 28July 2. Trans Europ Orthod Soc:59-68.

Salido EC, Yen PH, Koprivnikar K, Yu LC, Shapiro LJ (1992). The human enamel protein gene amelogenin is expressed from both the $X$ and the $Y$ chromosomes. Am I Hum Genet 50:303-316.

Smith $C$ (1974). Concordance in twins: methods and interpretation. Am I Hum Genet 26:454-466.

Townsend GC, Brown T (1978a). Inheritance of tooth size in Australian Aboriginals. Am ( Phys Anthropol 48:305-314.

Townsend GC, Brown T (1978b). Heritabilities of permanent tooth size. Am J Phys Anthropol 49:497-502. 